High-frequency dynamics and the spin-glass transition

This content has been downloaded from IOPscience. Please scroll down to see the full text. 1996 Europhys. Lett. 33489

(http://iopscience.iop.org/0295-5075/33/6/489)

View the table of contents for this issue, or go to the journal homepage for more

Download details:

IP Address: 131.215.70.231

This content was downloaded on 08/07/2014 at 15:54

Please note that terms and conditions apply. 
Europhys. Lett., 33 (6), pp. 489-494 (1996)

\title{
High-frequency dynamics and the spin-glass transition
}

\author{
D. Bitko ${ }^{1}$, N. Menon ${ }^{1}$, S. R. Nagel ${ }^{1}$, T. F. Rosenbaum ${ }^{1}$ and G. Aeprli ${ }^{2}$ \\ 1 The James Franck Institute and Department of Physics \\ The University of Chicago, Chicago, IL 60637, USA \\ 2 ATET Bell Laboratories, Murray Hill, NJ 07974, USA
}

(received 18 September 1995; accepted in final form 11 January 1996)

PACS. 75.50Lk - Spin glasses and other random magnets.

PACS. 75.40Gb - Dynamic properties (dynamic susceptibility, spin waves, spin diffusion, dynamic scaling, etc.).

PACS. 64.70Pf - Glass transitions.

\begin{abstract}
We identify two distinct regimes in the high-frequency response of the insulating, Ising spin-glass, $\mathrm{LiHo}_{0.167} \mathrm{Y}_{0.833} \mathrm{~F}_{4}$. The asymptotic high-frequency behavior of the imaginary part of the magnetic susceptibility becomes frequency independent as the spin-glass transition is approached: the shortest and the longest measured time scales both contain information about the actual phase transition. We compare our results to corresponding data on supercooled liquids.
\end{abstract}

Dynamical processes in glasses and spin glasses span an enormous range of time scales. In spin glasses, both the long-time behavior of the dynamical susceptibility and a divergent non-linear susceptibility provide clear signatures of the glass transition. In supercooled liquids, by contrast, the connections between the apparent divergence of relaxation times at finite temperature and a possible underlying phase transition are as yet unconfirmed.

Menon and Nagel [1] recently have explored the systematics of the high-frequency contributions to the dielectric susceptibility of simple liquids [2] approaching the glass transition. They identified two separate regimes at frequencies greater than the peak in the imaginary part of the susceptibility, and proposed a relationship between the asymptotic high-frequency behavior, the width of the dynamic response, and an actual divergent static susceptibility at the glass transition. It is our purpose in this letter to investigate the possibility of similar relationships in spin glasses where spectroscopic studies are sparser, but the evidence for critical behavior is stronger.

We chose for our studies the dipolar-coupled, Ising spin-glass, $\mathrm{LiHo}_{0.167} \mathrm{Y}_{0.833} \mathrm{~F}_{4} \cdot \mathrm{LiHo}_{x} \mathrm{Y}_{1-x} \mathrm{~F}_{4}$ is an isostructural dilution series where magnetic $\mathrm{Ho}^{3+}$ and nonmagnetic $\mathrm{Y}^{3+}$ ions randomly occupy rare-earth $(\mathrm{R})$ sites in the $\mathrm{LiRF}_{4}$ lattice. The pure compound $(x=1)$ is a ferromagnet, with an essentially perfect mean-field transition [3] at $T_{\mathrm{c}}=1.53 \mathrm{~K}$. Sufficient dilution destroys any long-range order, and the disorder and frustration (arising from the mix of ferromagnetic and antiferromagnetic interactions in a dipolar-coupled system) combine to make an

(C) Les Editions de Physique 


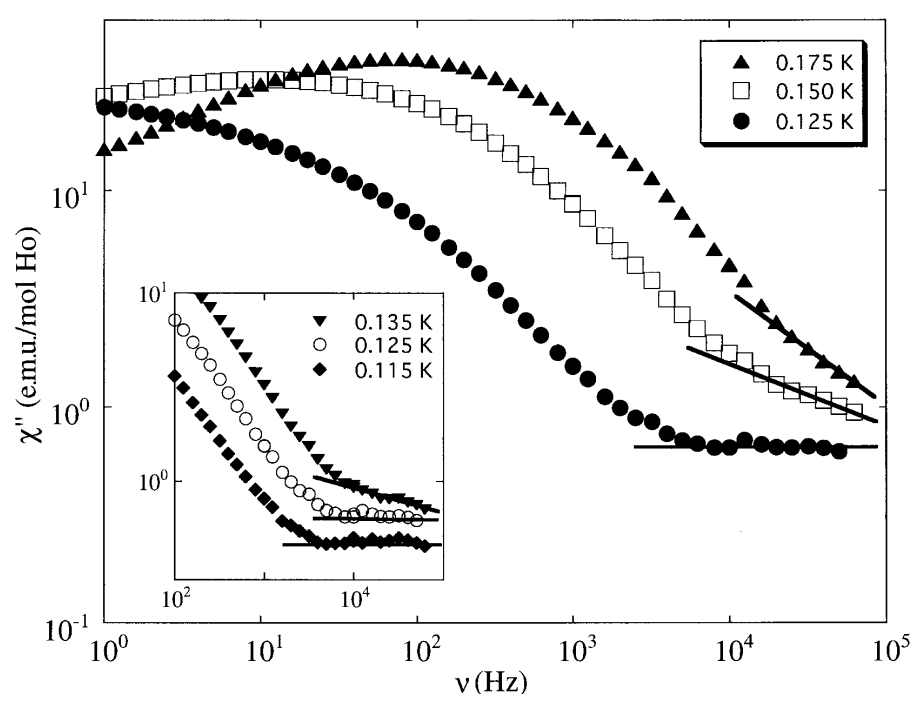

Fig. 1. - Log-log plot of the imaginary part of the magnetic susceptibility, $\chi^{\prime \prime}$, over five decades of frequency, $\nu$, for the Ising spin-glass at three temperatures, with $H_{\mathrm{t}}=0$ and $T_{\mathrm{g}}=0.13 \mathrm{~K}$. The power law in frequency just above the maximum in $\chi^{\prime \prime}(\nu)$ crosses over to a new power law at high $\nu$, and $\chi^{\prime \prime}(\nu)$ becomes frequency independent both the high- and low- $\nu$ limits for $T<T_{\mathrm{g}}$. Lines are guides to the eye. Inset: the high-frequency response bordering the spin-glass transition.

$x=0.167$ sample a spin-glass with $T_{\mathrm{g}}=0.13 \mathrm{~K}$ [4]. The crystals are insulating, which permits spectroscopy up to high frequency without limitations from eddy current heating. Moreover, the characteristic response times can be tuned in the laboratory through the application of a transverse magnetic field, $H_{\mathrm{t}}$. The transverse field mixes the eigenfunctions of the ground-state Ising doublet with the previously inaccessible excited states, leading to a rapid increase in the relaxation due to the existence of new tunneling models, and a complete suppression of spin-glass freezing at all $T$ by $H_{\mathrm{t}}=12 \mathrm{KOe}[5]$.

We suspended single crystals of $\mathrm{LiHo}_{0.167} \mathrm{Y}_{0.833} \mathrm{~F}_{4}$ of typical dimensions $(1.6 \times 1.6 \times 5) \mathrm{mm}^{3}$ from the mixing chamber of a helium dilution refrigerator inside the bore of a superconducting magnet with its Ising (long) axis oriented perpendicular to the main field direction. A trim coil, oriented parallel to the crystal's Ising axis, was used to compensate for any sample misalignment. We measured the magnetic susceptibility, $\chi(\nu)=\chi^{\prime}(\nu)+i \chi^{\prime \prime}(\nu)$, along the Ising axis over a frequency range $10^{0}-10^{5} \mathrm{~Hz}$ in a standard gradiometer configuration using a computer-based digital lock-in technique [6]. At the highest frequencies, we explicitly accounted for a weak non-linear response of the measuring coils due to a resonance at $\sim 10^{6} \mathrm{~Hz}$.

We plot in fig. 1 and $2 \chi^{\prime \prime}(\nu)$ at a series of temperatures which bracket the spin-glass transition, $T_{\mathrm{g}}\left(H_{\mathrm{t}}\right)$, with $T_{\mathrm{g}}(0)=0.13 \mathrm{~K}$ and $T_{\mathrm{g}}\left(H_{\mathrm{t}}=5 \mathrm{kOe}\right)=0.10 \mathrm{~K}$. Comparison of fig. 1 and 2 demonstrates that the application of a transverse field effectively shifts the spectral response to lower temperatures within our experimental window, while retaining the salient qualitative features. As in supercooled liquids [1], the general distribution function of relaxation times which gives rise to the measured susceptibility can be effectively described by three regimes: one power law in frequency, with positive exponent, below the peak; another power law, with negative exponent, just above the peak; and, finally, a third power law in the high- $\nu$ limit. Strikingly, the exponent of the high-frequency power law approaches zero ( $\chi^{\prime \prime}$ becomes frequency independent) for $T<T_{\mathrm{g}}\left(H_{\mathrm{t}}\right)$. This effect can be seen most clearly in the insets to fig. 1 and 2 , where we have magnified the high-frequency response in 


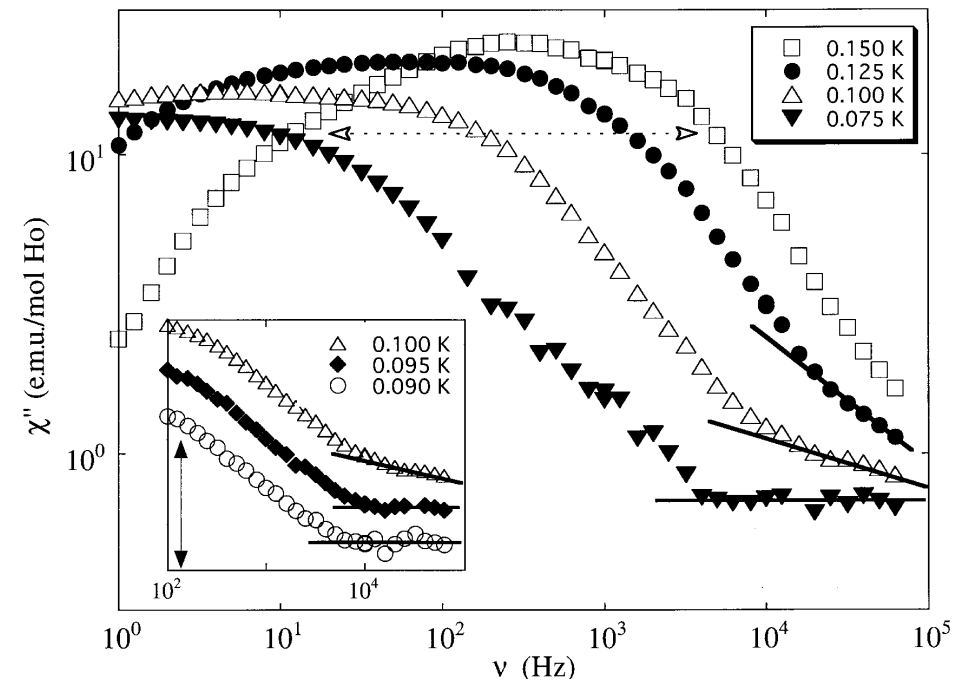

Fig. 2. - Counterpart to fig. 1 in a transverse magnetic field, $H_{\mathrm{t}}=5 \mathrm{kOe}$, with $T_{\mathrm{g}}\left(H_{\mathrm{t}}\right)=0.1 \mathrm{~K}$. The spectroscopic response retains three regimes in $\nu$, but has shifted to higher frequency. The horizontal arrow indicates the FWHM at $T=0.150 \mathrm{~K}$. Inset: the high-frequency response bordering the spin-glass transition. The data have been offset vertically for clarity. Vertical arrow corresponds to one decade in $\chi^{\prime \prime}$.

the immediate vicinity of $T_{\mathrm{g}}\left(H_{\mathrm{t}}\right)$. As expected, the transition between the effective power laws above the peak moves systematically to lower frequency with decreasing $T$ at fixed $H_{\mathrm{t}}$. A simple crossover between low- and high-frequency behavior does not adequately describe the data; the intermediate-frequency response remains robust, with a well-defined $\nu^{-0.70 \pm 0.05}$ dependence at all $T$.

The frequency independence of $\chi^{\prime \prime}$ at as asymptotically high $\nu$ is echoed by its saturation and frequency independence at low $\nu$. A flat spectrum in $\chi^{\prime \prime}$ as $\nu \rightarrow 0$ corresponds to $1 / f$ ( $f=$ frequency) noise in the magnetization by the fluctuation-dissipation theorem, and has been used to define the spin-glass state [5], [7], [8]. The asymptotic high-frequency response of $\chi^{\prime \prime}(\nu)$ also reflects the approach to $T_{\mathrm{g}}\left(H_{\mathrm{t}}\right)$, indicating that the shortest and longest time scales in the system contain similar information about the critical behavior. As with the general phenomenon of critical slowing-down at the approach to a phase transition, $h \nu \ll k T$ even at our highest frequencies.

We reproduce in fig. 3 the phase diagram for $\mathrm{LiHo}_{0.167} \mathrm{Y}_{0.833} \mathrm{~F}_{4}$ in transverse field [5]. The model Hamiltonian for $N$ Ising spins with random exchange $J_{i j}$ and transverse field $\Gamma$,

$$
H=-\sum_{i, j}^{N} J_{i j} \sigma_{i}^{z} \sigma_{j}^{z}-\Gamma \sum_{i}^{N} \sigma_{i}^{x},
$$

accurately describes the physics, where the $\sigma$ 's are Pauli spin matrices and $\Gamma \sim H_{\mathrm{t}}{ }^{2}$ at low $H_{\mathrm{t}}$. The open diamonds and squares are derived from conventional measurements of the onset of frequency independence of $\chi^{\prime \prime}(\nu \rightarrow 0)$ and the divergence of the non-linear susceptibility $\chi_{3}^{\prime}$, respectively [5]. The filled circles are obtained from estimating the onset of frequency independence of $\chi^{\prime \prime}(\nu)$ in the high-frequency limit (fig. 1 and 2). These points track the demarcation between paramagnet and spin-glass derived in the static limit, providing a stringent test of the relationship between the low- and high-frequency limits of the spectroscopic response. 


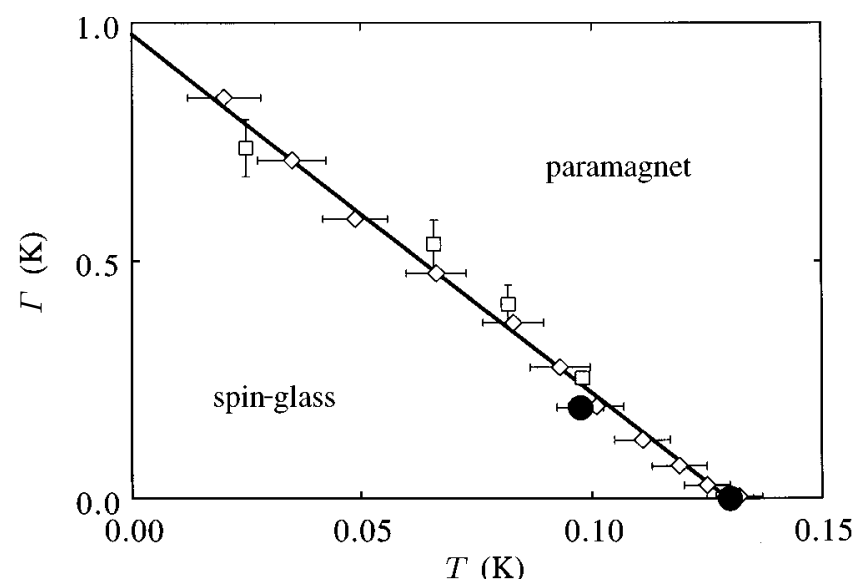

Fig. 3. - Phase diagram for the Ising spin-glass in transverse field (eq. (1)) determined from the asymptotic low-frequency behavior (open diamonds), the asymptotic high-frequency response (filled circles), and the divergence of the non-linear susceptibility (open squares) [5].

A divergence of the static dielectric susceptibility at the structural glass transition is posited to occur when the width $w$ of the imaginary part of the dynamic response approaches a finite value, $w^{*} \approx 2.6$, as extrapolated from temperatures well above the transition [1]. Here, $w$ is normalized to the 1.14 decades full width at half-maximum (FWHM) for a single relaxation time Debye peak. Certain spin-glass models [9] predict a comparable limiting width for the magnetic susceptibility, $w^{*} \approx 2.85$, reflected in dynamical studies of a short-range Ising spin-glass [10]. We find a related signature in our data, extended down to $10^{-1} \mathrm{~Hz}$ for this purpose [11]. We plot in fig. 4 the normalized full width at half-maximum of $\chi^{\prime \prime}(\nu)$ as a function of $H_{\mathrm{t}}$ at a series of temperatures (see, e.g., the demarcation of the FWHM for the $T=0.150 \mathrm{~K}$ data in fig. 2). The approach to frequency independence in the low-frequency part of the spectrum indicates that the width gets arbitrarily large as $T_{\mathrm{g}}$ is approached [5]. If we follow the behavior at fixed temperature as a function of decreasing field, we see a distinct change in curvature at $w^{*} \sim 3$. The solid symbols are all for data taken in the paramagnetic phase at temperatures above $T_{\mathrm{g}}\left(H_{\mathrm{t}}\right)$. The open symbols show the two lowest temperatures, $T=0.125$ and $0.100 \mathrm{~K}$, where the sample goes into the spin-glass phase as $H_{\mathrm{t}}$ is decreased. For these two temperatures, the transition occurs near to the value $w^{*}=3$, where the curves show a dramatic change in curvature. It is also possible to view the data of fig. 4 as a function of decreasing $T$ at fixed $H_{\mathrm{t}}$. In this case, it is apparent that the change in curvature leads to a rapid broadening of the width once $w^{*} \sim 3$ has been reached.

There remain significant differences between the dynamic responses of glasses and spinglasses: i) In this spin-glass, the high-frequency edge of the peak in $\chi^{\prime \prime}(\nu)$ remains at finite frequency even as $T_{\mathrm{g}}$ is approached. (Although the peak in $\chi^{\prime \prime}(\nu)$ broadens greatly as $T_{\mathrm{g}}$ is approached, the structure shown in fig. 1 and 2 remains in our experimental frequency window.) In glasses, the peak frequency going to zero is the signature of the glass transition. ii) At intermediate frequencies, the (first) power law above the peak in the imaginary part of the susceptibility is correlated with the normalized width $w$ in glasses, but remains fixed in spin-glasses. iii) The extrapolated value of $w$ approaches a finite limit at the glass transition, but may increase without bound at the spin-glass transition. We will address the implications for the static susceptibility, $\chi^{\prime}(0)$, of the temperature dependence of the width in a future 


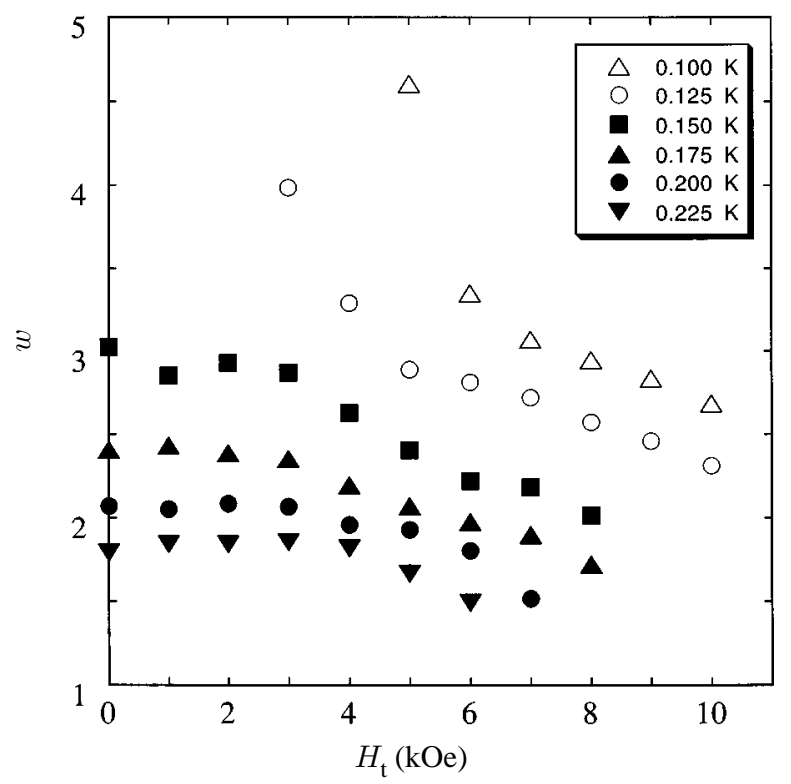

Fig. 4. - The normalized width, $w$, of the spectral response (see fig. 1) changes dramatically at $w^{*} \sim 3$, in accord with data on supercooled liquids [1] and certain spin-glass models [9]. Solid symbol are for data taken in the paramagnet; open symbol show the two lowest temperatures where the sample freezes into a spin-glass at small $H_{\mathrm{t}}$.

publication [12]; and iv) The $\nu \rightarrow 0$ power law below the maximum in the susceptibility is fixed in glasses, but is correlated with the high-frequency behavior (and $T_{\mathrm{g}}$ ) in spin-glasses.

We have shown that a representative spin-glass, like supercooled liquids [1], [2] and like plastic crystals [13], exhibits two separate regimes of behavior above the peak in $\chi^{\prime \prime}(\nu)$. The microscopic origin of this high-frequency behavior remains unclear, but the fact that it is found in three very different kinds of disordered materials points to its general importance. Furthermore, both the form of the magnetic/dielectric susceptibility at high frequency and the width of the spectroscopic response appear to contain information about the transition itself in at least two of these three classes of disordered systems. Attempts to develop a general framework [14] for systems with annealed and quenched disorder may profit from studying short-time as well as long-time dynamics.

We are indebted to W. Wu for his many contributions to this project. The work at the University of Chicago was supported primarily by the MRSEC Program of the National Science Foundation under Award No. DMR-9400379.

\section{REFERENCES}

[1] Menon N. and Nagel S. R., Phys. Rev. Lett., 74 (1995) 1230.

[2] Dixon P. K. et al., Phys. Rev. Lett., 65 (1990) 1108; Wu L. et al., J. Non-Cryst. Solids, 131 (1991) 32.

[3] Griffin J. A., Huster M. and Folweiler R. J., Phys. Rev. B, 22 (1980) 4370.

[4] Reich D. H. et al., Phys. Rev. B, 42 (1990) 4631. 
[5] Wu W. et al., Phys. Rev. Lett., 67 (1991) 2076; 71 (1993) 1919.

[6] Birge N. O. and Nagel S. R., Rev. Sci. Instrum., 58 (1987) 1164; Dixon P. K. and Wu L., Rev. Sci. Instrum., 60 (1989) 3329.

[7] Paulsen C. C., Williamson S. J. and Maletta H., Phys. Rev. Lett., 59 (1987) 128.

[8] Ocio M., Bouchiat H. and Monod P., J. Phys. (Paris), 46 (1985) L647; Fisher D. and Huse D., Phys. Rev. Lett., 56 (1986) 1601.

[9] Ogielski A. T., Phys. Rev. B, 32 (1985) 7384; Campbell I. A. et al., J. Phys. C, 20 (1987) L47.

[10] Gunnarsson K. et al., Phys. Rev. Lett., 61 (1988) 754.

[11] For an extended discussion of the lower-frequency response, see Wu W., PhD Thesis, University of Chicago (1993), unpublished.

[12] Menon N., Bitko D., Nagel S. R., Rosenbaum T. F. and Coppersmith S. N., to be published.

[13] Leslie-Pelecky D. L. and Birge N. O., Phys. Rev. Lett., 72 (1994) 1232.

[14] Parisi G., preprint; Franz S. and Hertz J., Phys. Rev. Lett., 74 (1995) 2114. 